\title{
Recurrent scoliosis one year after surgical correction
}

\author{
P. J. van Luijk $\cdot$ F. de Nies
}

Received: 23 December 2009/Revised: 6 June 2010/Accepted: 18 July 2010/Published online: 31 July 2010

(C) The Author(s) 2010. This article is published with open access at Springerlink.com

\begin{abstract}
A year after anterolateral spondylodesis for progressive scoliosis, the patient showed a flexion gait pattern with recurrent deformity, due to late infection. Surgical debridement resolved all symptoms. Whereas most postoperative infections occur after posterior spondylodesis and present with back pain and mild increase of infection parameters, late infection after anterolateral approach is rare. In this case the patient did not present with the classic symptoms.
\end{abstract}

Keywords Infection - Scoliosis - Anterolateral spondylodesis $\cdot$ Surgical debridement

\section{Case}

A 13-year-old girl attended our hospital with mild low back pain and a left-sided primarily lumbar scoliosis of $28^{\circ}$ Cobb angle. Brace treatment was started, but unfortunately the curves progressed in time. At the age of 15 the Cobb angle was over $50^{\circ}$. An anterolateral correction and fusion from Th12 to L3 was performed by left lumbotomy, using Monarch (Depuy Spine) instrumentation and iliac crest bone graft (Fig. 1). After a quick and uncomplicated

\footnotetext{
P. J. van Luijk · F. de Nies

Onze Lieve Vrouwe Gasthuis, Postbus 95500,

1090 HM Amsterdam, The Netherlands

P. J. van Luijk

Anthony Fokkerweg 57, 2316 ZG Leiden, The Netherlands

Present Address:

P. J. van Luijk ( $\square)$

Vijfmeilaan 8c, 2321 RL Leiden, The Netherlands

e-mail: petervanluijk@gmail.com
}

recovery and a correction up to less than $20^{\circ}$ she was discharged from the hospital in 5 days.

At routine intervals of 3 months she attended the outpatient department, the rehabilitation program was undisturbed and the curves remained unchanged.

However, 1 year after surgery, she returned somewhat earlier than expected, with a recurrent scoliosis, a significant left sided lateral deviation and flexion of the spine as well as a flexion contracture of the left hip. Pain was not the primary issue. Neither she nor we were able to redress the severe curve. BSE, CRP and WBC were within normal range. Besides this recurrent scoliosis below the instrumented level on plain X-rays and CT-scan, no complications were seen. The instrumented curve and screw position were unchanged and the fusion was solid.

On MRI a thickened psoas muscle was seen at the left side with significant oedema due to either infection or neoplasm. CT-guided cultures were taken, but no microorganisms were isolated. Histology reported non-specific inflammation, but not malignancy.

Over a period of time the general condition of the girl got worse with progression of the curve, lateral deviation and pain. Repeated blood tests now showed a raised BSE, CRP and WBC. Finally, almost 2 years after initial surgery, another MRI-scan revealed a large abscess around the screws and rods as well as in and underneath the left psoas muscle (Fig. 2). The hardware was removed, the abscess was drained and a thorough debridement was performed. This time the cultures showed $S$. aureus for which intravenous antibiotics were administered (Flucloxacilline $1,000 \mathrm{mg}, 6$ times daily).

She recovered very well, without wound healing problems and the infection parameters returned to normal values. The serious derangement of the posture resolved completely. 


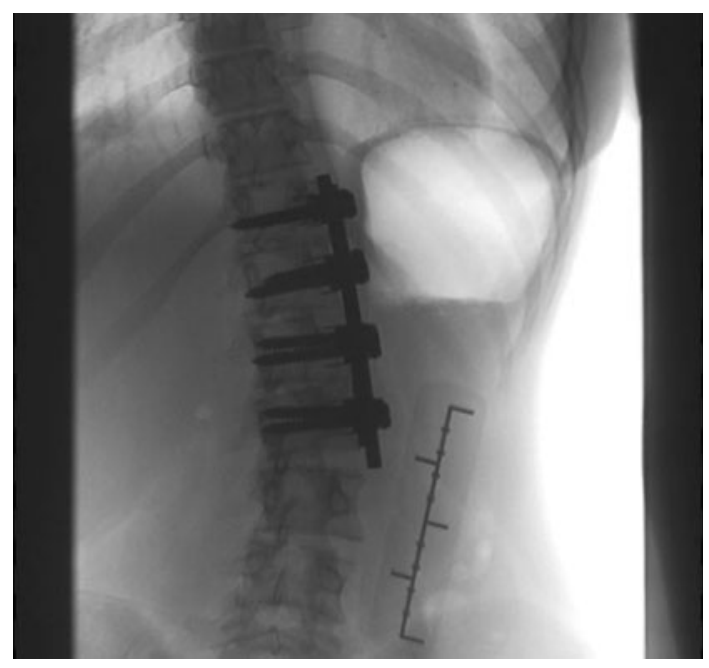

Fig. 1 The corrected spinal column

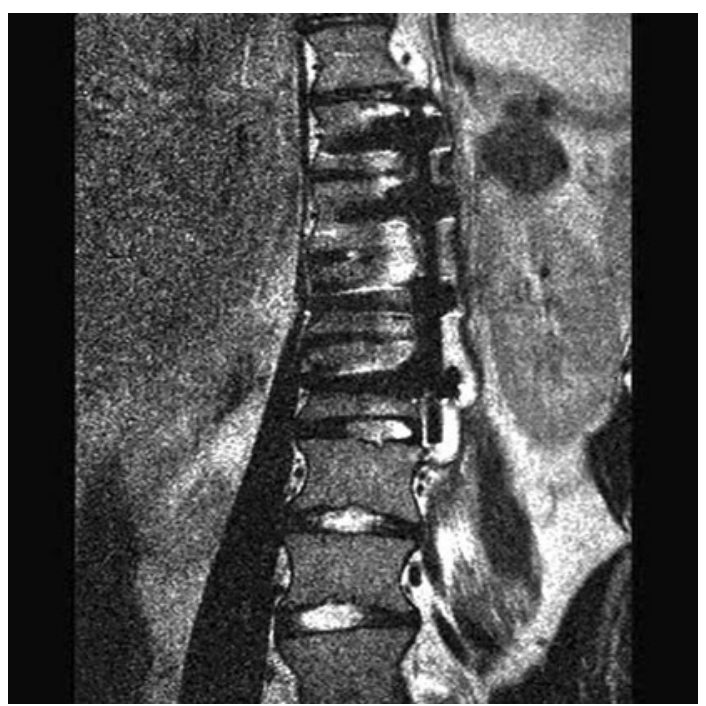

Fig. 2 The infected region. Visible around instrumentation and left psoas muscle

\section{Discussion}

In scoliosis surgery infections are rare, but well described, usually occurring in the direct post-operative period, incidentally as a late infection one or more years after initial surgery. In literature $[1,2]$ these late infections are thought to be localised around a small part of the hardware, presenting with swelling, local back pain and slightly if at all raised infection parameters (ESR, WBC and CRP). All reported cases followed surgery by posterior approach. Our patient, however, presented a year after anterolateral surgery with a severe left-side trunk shift and flexion contracture of the hip due to an extended psoas abscess, which was not appreciated in the initial radiological investigations. This is quite different from the localised infections near the hardware [1-3] after posterior approach. The epidural abscess as described by Choma [4] developed over a year after surgery, but this case was known with an early postoperative wound infection in contrast to our patient.

A late deep infection was taken into account, but although several cultures were taken, S. aureus was isolated only after surgical debridement, as recommended in literature $[1,2]$.

Conservative treatment alone (i.v. antibiotics) was not sufficient as to be expected in retrospect. Only after aggressive surgical intervention our patient fully recovered.

Anterior surgery is seldom associated with late lowgrade infection, at least no articles on this subject were found in literature. This is probably due to the richly vascularised structures covering the anterior lower spine. There was no specific reason for this previously perfectly healthy girl to develop a late/low-grade infection, so this must be considered a secondary, blood born infection.

\section{Conclusion}

Most late/low-grade infections in scoliosis correction described in literature follow posterior surgery and present with local back pain and swelling. We present a case of late, most likely secondary infection after anterolateral approach, with a recurrence of the scoliosis as the main symptom, which has not been described before. As in posterior approach surgical debridement and i.v. antibiotics are treatment of choice.

\section{Conflict of interest None.}

Open Access This article is distributed under the terms of the Creative Commons Attribution Noncommercial License which permits any noncommercial use, distribution, and reproduction in any medium, provided the original author(s) and source are credited.

\section{References}

1. Ho J (2007) Management of infection after instrumented posterior spine fusion in paediatric scoliosis. Spine 32(24):2739-2744

2. Rihn JA (2009) Infection after the surgical treatment of adolescent idiopathic scoliosis. Spine 33(3):289-294

3. Randall W (1997) Delayed infection after elective spinal instrumentation and fusion: a retrospective analysis of eight cases. Spine 22(20):2444-2450

4. Choma T (2008) Epidural abscess as a delayed complication of spinal instrumentation in scoliosis surgery. Spine 33(3):E76-E80 